\section{(1) \\ CrossMark}

\title{
Radial endobronchial ultrasound images for ground-glass opacity pulmonary lesions
}

\author{
Takehiro Izumo, Shinji Sasada, Christine Chavez, Yuji Matsumoto and \\ Takaaki Tsuchida
}

\author{
Affiliation: \\ Dept of Endoscopy, Respiratory Endoscopy Division, National Cancer Center Hospital, Tokyo, Japan. \\ Correspondence: \\ Takehiro Izumo, Dept of Endoscopy, Respiratory Endoscopy Division, National Cancer Center Hospital, 5-1-1 \\ Tsukiji Chuo-ku, Tokyo, 104-0045, Japan. \\ E-mail: drtake1118वgmail.com
}

ABSTRACT Radial endobronchial ultrasound (R-EBUS) is a useful tool for precise localisation of peripheral pulmonary lesions, but there have been no detailed reports about the use of R-EBUS images for ground-glass opacity (GGO).

The R-EBUS images of 116 patients with GGO, who were diagnosed as having adenocarcinoma by R-EBUS with a guide sheath (EBUS-GS), were compared with the respective chest computed tomography findings. In 103 patients, R-EBUS images were correlated with the histological surgical specimens.

R-EBUS images of GGO were identified based on the internal structure of the lesion and classified into two groups. Blizzard showed an enlarged, diffuse hyperintense acoustic shadow. Mixed blizzard showed a combination of blizzard and some diffuse heterogeneity with several hyperechoic dots and vessels. All pure GGO lesions (nine out of nine) were blizzard on R-EBUS. For part-solid GGOs, the percentage of mixed blizzard was inversely related to the amount of the GGO component. Histological findings from surgery revealed that all blizzard lesions were on the spectrum of adenocarcinoma in situ to well differentiated adenocarcinoma while majority (33 out of 64) of mixed blizzard lesions were moderately to poorly differentiated adenocarcinoma.

R-EBUS types are important to locate GGOs prior to transbronchial sampling with EBUS-GS.

$@$ ERSpublications

The classification of R-EBUS images is useful to decide the location of GGOs during transbronchial biopsy with R-EBUS http://ow.ly/FmCBR 


\section{Introduction}

Since the introduction of low-dose helical computed tomography (CT) scanning for lung cancer screening, the frequency of detecting pulmonary ground-glass opacity (GGO) has been about 20\% [1-3]. GGOs commonly represent a variety of diseases such as interstitial pneumonia, pulmonary lymphoproliferative disease, organising pneumonia, and pre-invasive, minimally invasive or moderately/poorly differentiated carcinoma [4]. Thus, definitive diagnosis is very important to choose an appropriate therapy. Surgical biopsy and CT-guided needle biopsy (CTNB) have been found to have a good diagnostic performance for GGOs, but come with accompanying risks such as pleural dissemination, pneumothorax and pulmonary haemorrhage, to name a few $[5,6]$.

Bronchoscopy offers a more safe diagnostic option [7], and guided techniques such as radial endobronchial ultrasound (EBUS), guide sheath, electromagnetic navigation, virtual bronchoscopy and ultra-thin bronchoscopy have improved the yield obtained for peripheral pulmonary lesions (PPLs) [8-10]. Among these, radial EBUS (R-EBUS) has the most data available with a diagnostic yield of $\geqslant 70 \%$ [8]. However, these past reports were based on data involving solid lesions.

Recently, transbronchial biopsy for GGO through EBUS with a guide sheath (EBUS-GS) has been reported $[11,12]$. The yield is lower compared with surgical biopsy and CTNB for GGOs and to previous research on bronchoscopy for solid PPLs. Nevertheless, these studies highlighted the importance of confirming the location of a GGO prior to biopsy. We previously reported that GGO, especially the pure type, usually demonstrates a subtle but noticeable increase in the intensity and radius of the whitish acoustic shadow of normal lung on EBUS [13-15]. To confirm the findings of these previous reports, the current study focused on the EBUS images of pure and part-solid GGO lesions and correlated these findings with thinsection CT findings and surgical histopathology. From its current use in solid lesions, we aim to extend the capability of EBUS to GGOs, because these lesions, especially part-solid GGOs, that do not resolve after 3 months are often malignant. The eventual goal is to improve the diagnostic yield of bronchoscopy for PPLs, including GGO.

\section{Materials and methods}

\section{Subjects}

The database of the Respiratory Endoscopy Division of the National Cancer Center, Tokyo, Japan was searched for patients who underwent EBUS-GS for PPL diagnosis between July 2012 and June 2014. Among 1134 patients seen during the study period there were 187 with GGOs that were suspected to be malignant. GGOs that were diagnosed as malignant by EBUS-GS were included in the study population.

The database and medical records of the study population were then reviewed and evaluated. This study was approved by the Institutional Review Board of the hospital, and all patients provided written informed consent.

\section{Methods and equipment}

All bronchoscopies were performed via the oral route under local anaesthesia with mild sedation (intravenous administration of midazolam). The bronchial route was planned by reviewing the chest CT images before bronchoscopy. Virtual bronchoscopic navigation/simulation systems (Ziostation2, Ziosoft Ltd, Tokyo, Japan; LungPoint, Bronchus Ltd, Mountain View, CA, USA; or Bf-Navi, Olympus Ltd, Toyko, Japan) were used to detect the bronchial route to the target lesions.

A PPL was defined as an abnormal growth surrounded by normal pulmonary parenchyma and was bronchoscopically invisible. In addition, there was no lymphadenopathy, atelectasis or effusion. Upon reaching the target bronchus, the guide sheath together with an ultrasound probe was inserted through the working channel of the scope and advanced towards the PPL under fluoroscopic guidance [16]. R-EBUS scanning was performed while manipulating the probe until the lesion was localised by the corresponding EBUS image. The biggest area on the EBUS image was captured and analysed. After confirming the location of the PPL, the probe was removed while keeping the guide sheath in place for the usual transbronchial sampling using brush, forceps and transbronchial needle aspiration under fluoroscopic guidance [17]. Cytology and histology specimens were sent for pathological examination.

\section{$R$-EBUS and guide sheath equipment}

R-EBUS was performed using an endoscopic ultrasound system (EU-ME30 and EU-ME1; Olympus Ltd) equipped with R-EBUS probes measuring $2.0 \mathrm{~mm}$ (UM-S20-20R; Olympus Ltd) or $1.4 \mathrm{~mm}$ (UM-S20-17S; Olympus Ltd) in diameter. The guide sheath used measured $2.55 \mathrm{~mm}$ (K-203 GS Kit; Olympus, Tokyo, Japan) or $1.95 \mathrm{~mm}$ in diameter (K-201 GS Kit; Olympus, Tokyo Japan). The ultrasound settings were constant for all the procedures. 


\section{Study variables}

Data collected were the PPL characteristics from the CT scan and R-EBUS image, and pathological findings on the surgical specimen.

\section{CT scan characteristics}

Every patient had a $5 \mathrm{~mm}$-slice chest CT scan carried out within 4 weeks of the procedure and an additional 1-mm slice chest CT scan using an 80-detector rows CT (Aquillion PRIME; Toshiba, Tokyo, Japan). Images were displayed with a lung window setting (centre: $-600 \mathrm{H}$; width: $1500 \mathrm{H}$ ). The PPLs were characterised based on the percentage of the GGO component (pure GGO, part-solid with $>75 \%$ GGO, part-solid with $>50-75 \%$ GGO, part-solid with $25-50 \%$ GGO, part-solid with $<25 \%$ GGO). A pure GGO was defined as a lesion with no solid portion.

\section{R-EBUS images}

The R-EBUS images of GGOs were named blizzard or mixed blizzard. This description formed the basis for the following proposed classification of R-EBUS types.

\section{Blizzard sign}

The blizzard sign was defined as a subtle, but noticeable increase in the intensity and radius of the whitish acoustic shadow while scanning from normal lung tissue (fig. 1a) to the ground-glass area. This change in the ultrasound signal is similar to a whiteout and has a relatively larger radius from the centre of the probe compared with the R-EBUS snowstorm appearance generated while scanning the area of normal alveolar tissue. On meticulous inspection, the details that constitute the acoustic signal are relatively thick and crude (fig. 1b) compared with the details that constitute the refined snowstorm appearance of normal lung tissue [13-15].

\section{Mixed blizzard sign}

In the mixed blizzard sign (fig. 1c) the internal echo of the lesions demonstrated diffuse heterogeneity with several hyperechoic dots, linear arcs and vessels that were distributed irregularly or combined with the blizzard sign described in the previous section.

\section{Statistical analysis}

Descriptive statistics are presented as frequency, percentage and median (range). Correlation of study variables were performed with EZR (Saitama Medical Center, Jichi Medical University; www.jichi.ac.jp/ saitama-sct/SaitamaHP.files/statmed.html), a graphical user interface for R (version 2.13.0, The R Project for Statistical Computing; http://www.r-project.org) and a modified version of $\mathrm{R}$ commander (version $1.8-4)$.

\section{Results}

There were a total of 116 patients diagnosed with lung adenocarcinoma by EBUS-GS. The baseline characteristics are summarised in table 1. 103 out of 116 patients underwent surgical resection. The remaining patients either opted for follow-up $(n=6)$, or were poor surgical candidates and instead
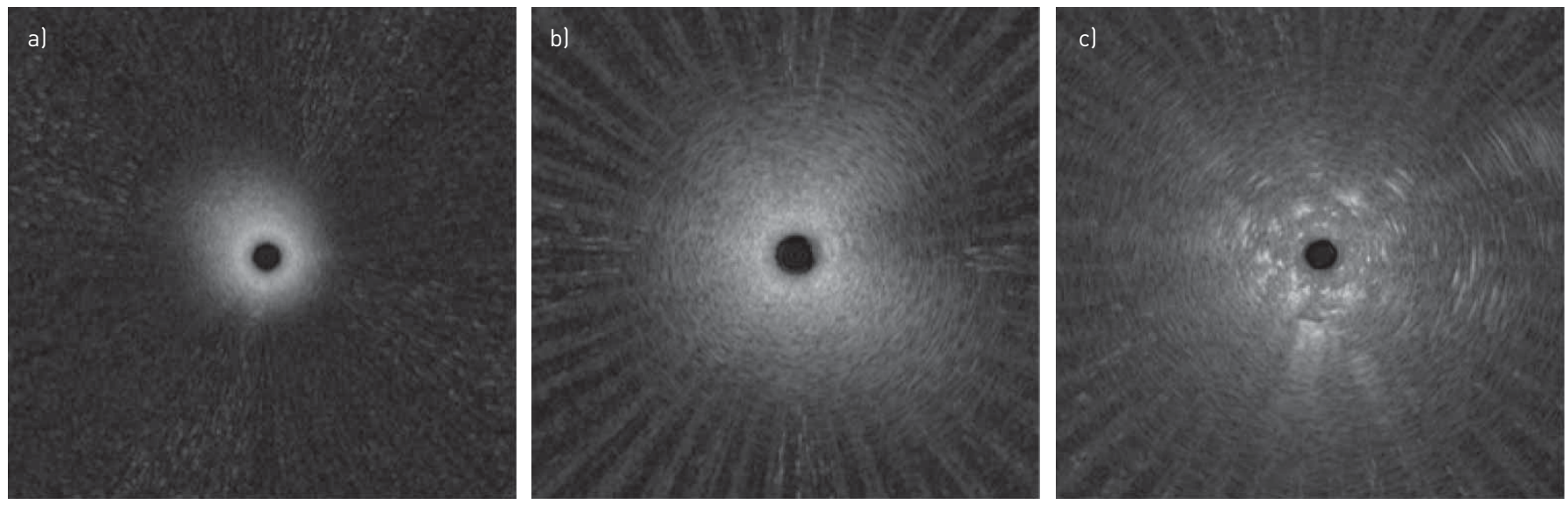

FIGURE 1 Representative radial endobronchial ultrasound images of normal lung and ground-glass opacity pulmonary lesions. a) Normal lung. b) A subtle, but noticeable increase in the intensity and radius of the whitish acoustic shadow (blizzard sign). c) Mixed blizzard sign, a diffusely heterogenous acoustic shadow (with some hyperechoic dots, linear arcs and vessels) that is distributed irregularly within a blizzard. 


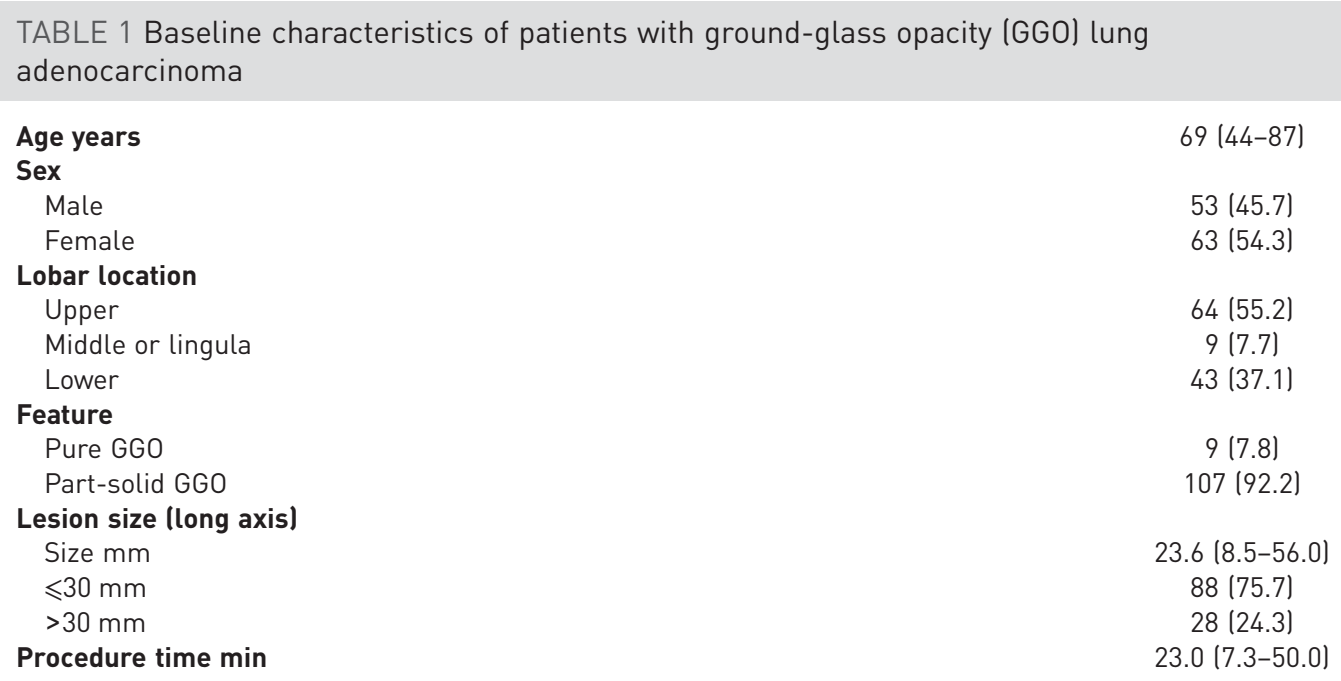

Data are presented as median (range) or $\mathrm{n}(\%) . \mathrm{n}=116$.

underwent radiation therapy $(n=5)$ or chemotherapy $(n=2)$. Of these 13 patients, five patients had R-EBUS images with a blizzard sign and eight patients had mixed blizzard sign.

Correlation between chest thin-section CT findings and R-EBUS types for GGO

Table 2 shows that the correlation between chest thin-section CT findings and R-EBUS types for GGO lesions diagnosed by EBUS-GS.

Blizzard was found in all pure GGO lesions (nine (100\%) out of nine), in six (85.7\%) out of seven part-solid lesions with $>75 \%$ GGO, in $15(51.7 \%)$ out of 29 part-solid lesions with $>50-75 \%$ GGO, in 11 $(31.4 \%)$ out of 35 part-solid lesions with $25-50 \%$ GGO, and in three (8.3\%) of 36 part-solid lesions with $<25 \%$ GGO. Blizzard sign on the R-EBUS image was consistently observed in lesions with more GGO component.

Mixed blizzard was found in one (14.3\%) out of seven part-solid lesions with $>75 \%$ GGO, in 14 (48.3\%) out of 29 part-solid lesions with $>50-75 \%$ GGO, in 24 (68.6\%) out of 35 part-solid lesions with $25-50 \%$ GGO, and in $33(91.7 \%)$ out of 36 part-solid lesions with $<25 \%$ GGO. The mixed blizzard sign on the R-EBUS image was not found in any pure GGO lesion, but was consistently observed in lesions with a larger proportion of solid component.

Correlation between R-EBUS types and surgical histology for GGO

Table 3 shows the correlation between R-EBUS types and histopathology results of surgically resected GGO. All blizzard lesions were on the spectrum of adenocarcinoma in situ to well-differentiated adenocarcinoma, while the majority of mixed blizzard lesions were well-differentiated invasive lepidic-predominant and moderately to poorly differentiated adenocarcinoma.

Representative cases of blizzard and mixed blizzard lesions are shown in figures 2 and 3, respectively.

\section{Discussion}

Several publications have reported about the utility of R-EBUS with/without a guide sheath for PPLs, although most of them described solid lesions $[8,18]$. A few were concerned with the diagnostic yield for GGOs by EBUS-GS $[11,12]$, but there were no reports on R-EBUS images of GGOs, especially in relation to thin-section CT findings and histopathology. To our knowledge, this study is the first to demonstrate the typical types of R-EBUS images for GGOs secondary to adenocarcinoma.

A previous study has already described the internal structure of solid PPLs as visualised by EBUS and correlated these findings with the histopathology of thoracic malignancy and benign cases [19]. The study reported in detail the R-EBUS images of peripheral lesions, focusing on the homogeneity of the internal echo image, the patency of the internal blood vessels and the presence of internal air space. Obtaining an EBUS image is crucial to confirm the location of the target biopsy site and pulmonary physicians have used R-EBUS image classification to diagnose PPLs successfully [19]. 
TABLE 2 Correlation between chest thin-section CT findings and radial EBUS types for GGO lesions diagnosed by EBUS-GS

\begin{tabular}{|c|c|c|c|c|c|}
\hline & \multicolumn{5}{|c|}{ Thin-section CT findings } \\
\hline Subjects $\mathrm{n}$ & 9 & 7 & 29 & 35 & 36 \\
\hline \multicolumn{6}{|l|}{ EBUS types } \\
\hline Blizzard $^{\#}$ & $100(9 / 9)$ & $85.7(6 / 7)$ & $51.7(15 / 29)$ & $31.4(11 / 35)$ & $8.3(3 / 36)$ \\
\hline Mixed blizzard" & $0(0 / 9)$ & $14.3(1 / 7)$ & $48.3(14 / 29)$ & $68.6(24 / 35)$ & $91.7(33 / 36)$ \\
\hline
\end{tabular}

Data are presented as \% $(n / N)$, unless otherwise stated. Total $n=116$. CT: computed tomography; EBUS: endobronchial ultrasound; GGO: ground-glass opacity; EBUS-GS: EBUS with a guide sheath. " $: n=44 ;{ }^{\text {ๆl: }} n=72$.

Until now, detecting the location of GGOs has been a challenge because the histopathological changes in a GGO lesion, especially pure GGO, are minimal and cannot be perceived obviously by R-EBUS [11]. Recently we reported the typical R-EBUS images for pure GGO [13-15]. In fact, 43 of the cases in the present study were also included in these prior publications. In these reports, we described that the typical snowstorm appearance was generated as the ultrasound probe was in the area of normal lung tissue, but that as the probe was inserted more distally to the intended lung segment, a subtle but noticeable enhancement and increase in radius of the snowstorm appearance was observed. Although not yet clear, we hypothesise that the mechanism behind this change is diffraction phenomenon. Pure and GGO-dominant lesions demonstrate this pattern on R-EBUS (blizzard) and this could be explained by the large amount of residual air in the intact alveoli (without stromal invasion).

For part-solid GGO, a diffusely heterogenous acoustic shadow (with some hyperechoic dots, linear arcs and vessels) that is distributed irregularly within a blizzard was observed during R-EBUS scanning. This signal, mixed blizzard, may represent tumour invasion beyond the alveolar spaces, which usually happens when a GGO lesion develops a solid component [20]. The ultrasound image can be easily recognised because it appears hypoechoic compared with the snowstorm appearance of normal lung and can be explained by the difference in the medium through which the ultrasound wave was propagated (i.e., air-filled alveolar space versus alveolar space with less or no air). The GGO component that usually surrounds the periphery of a part-solid lesion corresponds to the area that generates a blizzard signal in the R-EBUS image.

It is important to note that the R-EBUS image depends on which part of the lesion the probe is in. EBUS generates an acoustic shadow from the area the probe touches. If the probe is in a bronchus that is close to

TABLE 3 Correlation between radial EBUS images and histology of surgically resected GGO

\begin{tabular}{lcc} 
& \multicolumn{2}{c}{ EBUS types } \\
\cline { 2 - 3 } & Blizzard & Mixed blizzard \\
\hline Subjects & 39 & 64 \\
Surgical diagnosis & & \\
Adenocarcinoma in situ & 1 & 2 \\
Minimally invasive adenocarcinoma & 9 & 16 \\
Well differentiated invasive lepidic predominant & 18 & 9 \\
Well differentiated invasive papillary predominant & 8 & 1 \\
Well differentiated invasive acinar predominant & 2 & 3 \\
Well differentiated mucinous (formerly mucinous BAC with invasion) & 1 & 1 \\
Moderately differentiated invasive lepidic predominant & 0 & 24 \\
Moderately differentiated invasive papillary predominant & 0 & 2 \\
Moderately differentiated invasive micropapillary predominant & 0 & 3 \\
Moderately differentiated invasive acinar predominant & 0 & 1 \\
Moderately differentiated mucinous (formerly mucinous BAC with invasion) & 0 & 2 \\
Poorly differentiated invasive solid predominant & 0 &
\end{tabular}

Data are presented as $n$. Total $n=103$. EBUS: endobronchial ultrasound; GGO: ground-glass opacity; BAC: bronchioloalveolar carcinoma. 

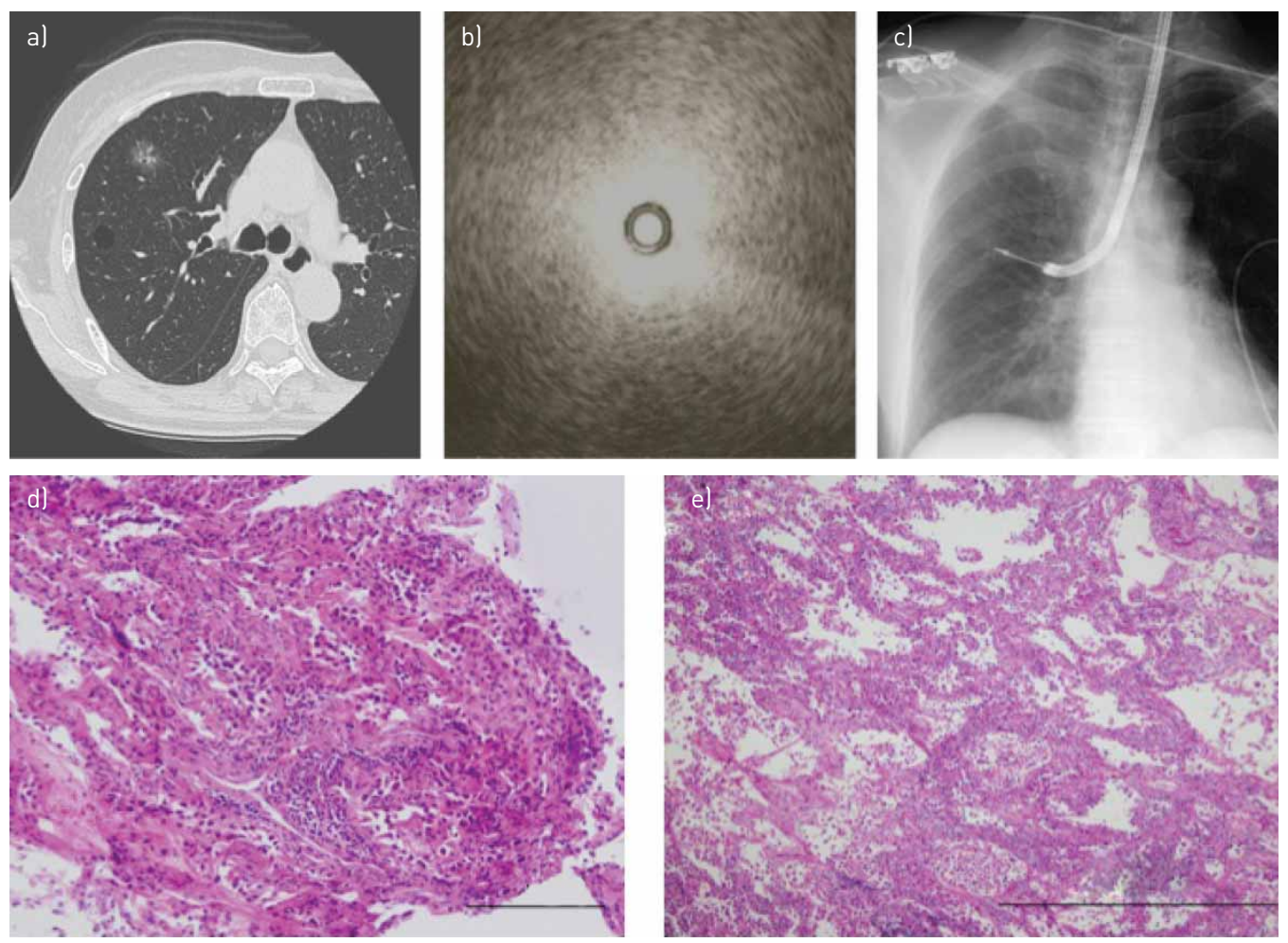

FIGURE 2 A representative case in a 74-year-old woman with pure ground-glass opacity (GGO) in the right upper lobe. a) Thin-section computed tomography scan showing a pure GGO in the right S3b. b) Radial endobronchial ultrasound (EBUS) showed a subtle but noticeable increase in the intensity and radius of the whitish acoustic shadow (blizzard sign). c) Real-time fluoroscopy image of the chest during radial EBUS scanning. d) Histopathological specimen from transbronchial biopsy showing cuboidal tumour cells lining the entrapped alveolar space (haematoxylin and eosin stain, original magnification $\times 100$ ). Scale $b a r=200 \mu \mathrm{m}$. e) Histopathological specimen from surgical biopsy showing a well-differentiated microinvasive adenocarcinoma (haematoxylin and eosin stain, original magnification $\times 40$ ). Scale bar $=1.0 \mathrm{~mm}$.

the GGO portion the R-EBUS image will show blizzard. By contrast, if the probe is in a bronchus that is close to the solid portion the R-EBUS image will show mixed blizzard. This could explain why, in this study, some part-solid lesions with $<50 \%$ GGO were classified as blizzard (table 2). Nevertheless, the point is that it is important to review on thin-section CT the bronchus that leads to the GGO lesion before every procedure in order to have an estimate of what type of R-EBUS image to look for during tumour localisation.

Selecting the correct location of PPLs by R-EBUS is essential for an accurate diagnosis by bronchoscopy, especially for malignant GGOs that are usually early stage cancers [20]. Moreover, precise localisation of a GGO by EBUS and the use of a guide sheath may be valuable for the development and improvement of novel therapeutic bronchoscopy procedures (radiofrequency ablation, etc.) for early-stage lung cancer $[21,22]$.

Some authors have suggested that a greater amount of GGO component in part-solid lesions corresponds to a better prognosis [23, 24]. From our correlation between the R-EBUS types and surgical histopathological results, we consider that blizzard may be the R-EBUS image for a low grade adenocarcinoma, while mixed blizzard may be the R-EBUS image for a less differentiated histology and, thus, a worse outcome.

This study has several limitations. First, it was a retrospective analysis in a single institute. Secondly, the bronchoscopy procedures were not performed by the same bronchoscopist and, as ultrasound is an operator dependent modality, the quality of the images captured during the procedure varied. Thirdly, the R-EBUS image depended on which part of the lesion the probe was in. Lastly, benign lesions were excluded because confirmation of definite diagnosis and accurate correlation between R-EBUS image and histopathology were difficult. For this reason, this study included only lung adenocarcinoma GGOs that were diagnosed by EBUS-GS. Prospective trials that take into account these confounding variables are recommended in the future. 

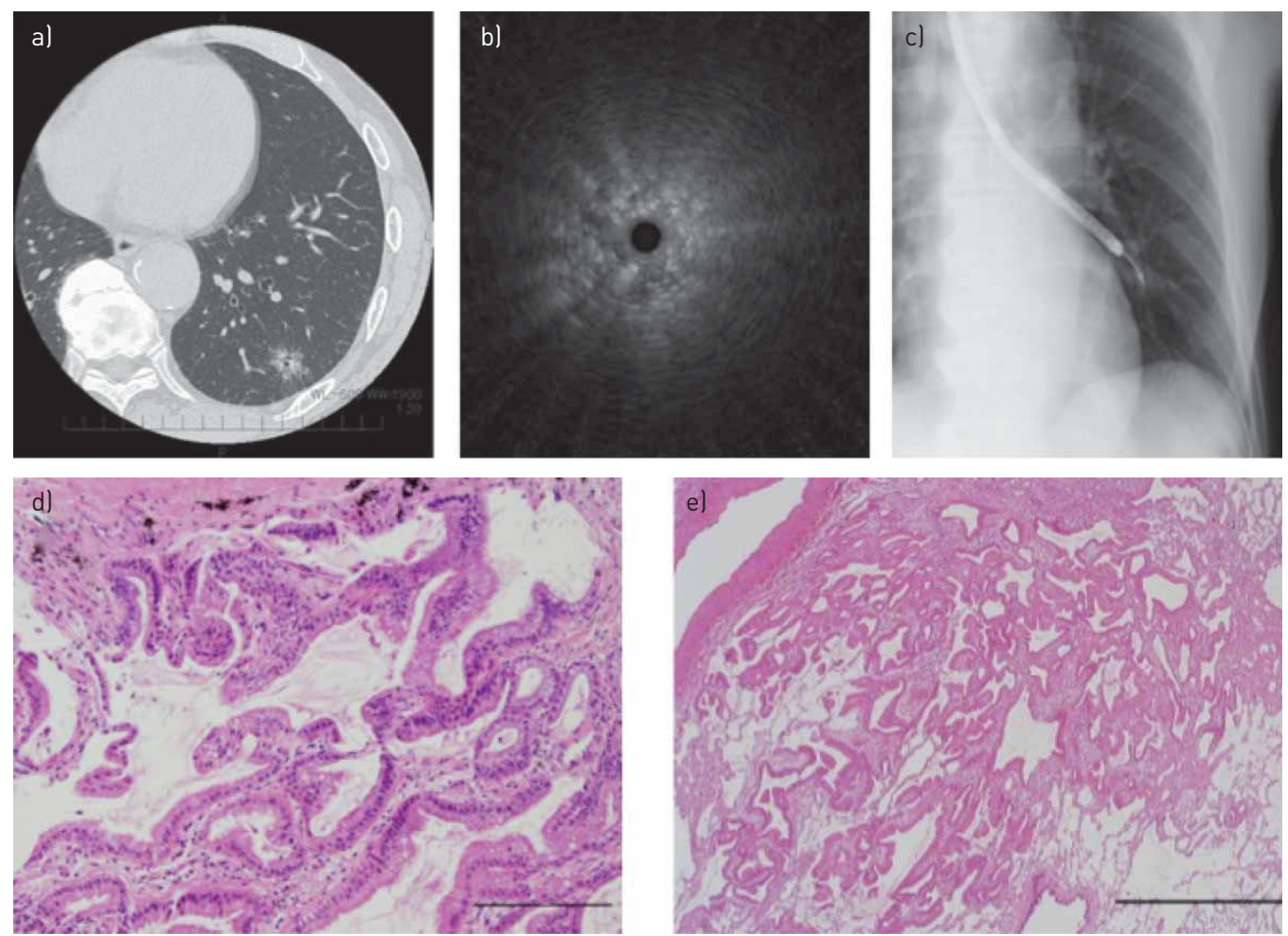

FIGURE 3 A representative case in a 80-year-old man with a part solid ground-glass opacity (GGO) in the left lower lobe. a) Thin-section computed tomography scan showing a part solid GGO in the left S10a. b) Radial endobronchial ultrasound (EBUS) showing mixed blizzard sign, a diffuse heterogeneity (with some hyperechoic dots, linear arcs and vessels) that is distributed irregularly within a blizzard. c) Real-time fluoroscopy image of the chest during radial EBUS scanning. d) Histopathological specimen from the transbronchial biopsy showing adenocarcinoma with lepidic growth pattern (haematoxylin and eosin stain, original magnification $\times 100$ ). Scale bar $=200 \mu \mathrm{m}$. e) Histopathological specimen from the surgical biopsy showing a well differentiated invasive lepidic-predominant adenocarcinoma (haematoxylin and eosin stain, original magnification $\times 40$ ). Scale bar $=1.0 \mathrm{~mm}$.

\section{Conclusions}

R-EBUS image classification of GGOs as blizzard and mixed blizzard can be one of the useful tools to detect the correct location of GGOs during EBUS-GS. In a part-solid GGO lesion, mixed blizzard sign may represent the area which is preferable to biopsy and, thus, is important to identify. Correlation of thin-section CT findings with each R-EBUS type may potentially affect accurate pathological diagnosis, leading to correct prognostication and, perhaps, application of novel therapy.

\section{References}

1 Henschke CI, Yankelevitz DF, Mirtcheva R, et al. CT screening for lung cancer: frequency and significance of part-solid and nonsolid nodules. AJR Am J Roentgenol 2002; 178: 1053-1057.

2 MacMahon H, Austin JH, Gamsu G, et al. Guidelines for management of small pulmonary nodules detected on CT scans: a statement from the Fleischner Society. Radiology 2005; 237: 395-400.

3 National Lung Screening Trial Research Team, Church TR, Black WC, et al. Results of initial low-dose computed tomographic screening for lung cancer. N Engl J Med 2013; 368: 1980-1991.

4 Lee HY, Lee KS. Ground-glass opacity nodules: histopathology, imaging evaluation, and clinical implications. J Thorac Imaging 2011; 26: 106-118.

5 Lu C-H, Hsiao C-H, Chang Y-C, et al. Percutaneous computed tomography-guided coaxial core biopsy for small pulmonary lesions with ground-glass attenuation. J Thorac Oncol 2012; 7: 143-150.

6 Inoue $\mathrm{D}$, Gobara $\mathrm{H}$, Hiraki $\mathrm{T}$, et al. CT fluoroscopy-guided cutting needle biopsy of focal pure ground-glass opacity lung lesions: diagnostic yield in 83 lesions. Eur J Radiol 2012; 81: 354-359.

7 Asano F, Aoe M, Ohsaki Y, et al. Bronchoscopic practice in Japan: a survey by the Japan Society for Respiratory Endoscopy in 2010. Respirology 2013; 18: 284-290.

8 Wang Memoli JS, Nietert PJ, Silvestri GA. Meta-analysis of guided bronchoscopy for the evaluation of the pulmonary nodule. Chest 2012; 142: 385-393.

9 Steinfort DP, Khor YH, Manser RL, et al. Radial probe endobronchial ultrasound for the diagnosis of peripheral lung cancer: systematic review and meta-analysis. Eur Respir J 2011; 37: 902-910.

10 Rivera MP, Mehta AC, Wahidi MM. Establishing the diagnosis of lung cancer: diagnosis and management of lung cancer, 3rd ed: American College of Chest Physicians evidence-based clinical practice guidelines. Chest 2013; 143: Suppl. 5, e142S-e165S. 
11 Izumo T, Sasada S, Chavez C, et al. The diagnostic utility of endobronchial ultrasonography with a guide sheath and tomosynthesis images for ground glass opacity pulmonary lesions. J Thorac Dis 2013; 5: 745-750.

12 Ikezawa Y, Sukoh N, Shinagawa N, et al. Endobronchial ultrasonography with a guide sheath for pure or mixed ground-glass opacity lesions. Respiration 2014; 88: 137-143.

13 Sasada S, Izumo T, Chavez C, et al. Blizzard sign as a specific endobronchial ultrasound image for ground glass opacity: a case report. Respir Med Case Reports 2014; 12: 19-21.

14 Chavez C, Sasada S, Izumo T, et al. Image-guided bronchoscopy for histopathologic diagnosis of pure ground glass opacity: a case report. $J$ Thorac Dis 2014; 6: E81-E84.

15 Sasada S, Izumo T, Chavez C, et al. A new middle-range diameter bronchoscope with large channel for transbronchial sampling of peripheral pulmonary lesions. Jpn J Clin Oncol 2014; 44: 826-834.

16 Katsurada M, Izumo T, Nagai Y, et al. The dose and risk factors for radiation exposure to medical staff during endobronchial ultrasonography with a guide sheath for peripheral pulmonary lesions under X-ray fluoroscopy. Jpn J Clin Oncol 2014; 44: 257-262.

17 Takai M, Izumo T, Chavez C, et al. Transbronchial needle aspiration through a guide sheath with endobronchial ultrasonography (GS-TBNA) for peripheral pulmonary lesions. Ann Thorac Cardiovasc Surg 2014; 20: 19-25.

18 Herth FJF, Eberhardt R, Becker HD, et al. Endobronchial ultrasound-guided transbronchial lung biopsy in fluoroscopically invisible solitary pulmonary nodules: a prospective trial. Chest 2006; 129: 147-150.

19 Kurimoto N, Murayama M, Yoshioka S, et al. Analysis of the internal structure of peripheral pulmonary lesions using endobronchial ultrasonography. Chest 2002; 122: 1887-1894.

20 Travis WD, Brambilla E, Noguchi M, et al. Diagnosis of lung cancer in small biopsies and cytology: implications of the 2011 International Association for the Study of Lung Cancer/American Thoracic Society/European Respiratory Society classification. Arch Pathol Lab Med 2013; 137: 668-684.

21 Tanabe $\mathrm{T}$, Koizumi $\mathrm{T}$, Tsushima $\mathrm{K}$, et al. Comparative study of three different catheters for CT imaging-bronchoscopy-guided radiofrequency ablation as a potential and novel interventional therapy for lung cancer. Chest 2010; 137: 890-897.

22 Tsushima K, Koizumi T, Tanabe T, et al. Bronchoscopy-guided radiofrequency ablation as a potential novel therapeutic tool. Eur Respir J 2007; 29: 1193-1200.

23 Suzuki K, Kusumoto M, Watanabe S, et al. Radiologic classification of small adenocarcinoma of the lung: radiologic-pathologic correlation and its prognostic impact. Ann Thorac Surg 2006; 81: 413-419.

24 Travis WD, Brambilla E, Noguchi M, et al. International Association for the Study of Lung Cancer/American Thoracic Society/European Respiratory Society international multidisciplinary classification of lung adenocarcinoma. J Thorac Oncol 2011; 6: 244-285. 